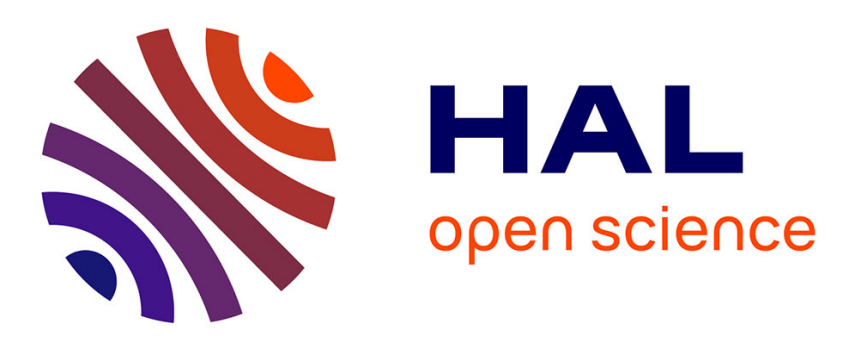

\title{
The fabrication of nanodiamond doped tellurite germanate glass with robust structure and its luminescence
}

Yayan Xu, Haiyong Gan, Zhanling Lu, Renguang Ye, Tanghan Chen, Junjie Zhang, Ying Tian, Shiqing Xu

\section{To cite this version:}

Yayan Xu, Haiyong Gan, Zhanling Lu, Renguang Ye, Tanghan Chen, et al.. The fabrication of nanodiamond doped tellurite germanate glass with robust structure and its luminescence. Journal of the American Ceramic Society, 2021, 104 (4), pp.1698-1706. 10.1111/jace.17569 . hal-03100291

\section{HAL Id: hal-03100291 \\ https://hal.science/hal-03100291}

Submitted on 12 Jan 2021

HAL is a multi-disciplinary open access archive for the deposit and dissemination of scientific research documents, whether they are published or not. The documents may come from teaching and research institutions in France or abroad, or from public or private research centers.
L'archive ouverte pluridisciplinaire HAL, est destinée au dépôt et à la diffusion de documents scientifiques de niveau recherche, publiés ou non, émanant des établissements d'enseignement et de recherche français ou étrangers, des laboratoires publics ou privés. 
DR YING TIAN (Orcid ID : 0000-0002-7842-6162)

Article type : Article

\section{The fabrication of nanodiamond doped tellurite germanate glass with robust structure and its luminescence}

Yayan Xu, ${ }^{1} \mid$ Haiyong Gan, ${ }^{2} \mid$ Zhanling Lu, ${ }^{3} \mid$ Renguang Ye, ${ }^{1,4} \mid$ Tanghan Chen, ${ }^{1} \mid$ Junjie Zhang, ${ }^{1} \mid$

Ying Tian, ${ }^{1, *} \mid$ Shiqing $\mathrm{Xu}^{1, *}$

${ }^{1}$ Institute of photoelectric materials and devices, China Jiliang University, Hangzhou 310018, PR China

${ }^{2}$ National Institute of Metrology, Beijing 100013, PR China

${ }^{3}$ School of Materials Science and Engineering, Zhengzhou University, Zhengzhou 450001, PR China

${ }^{4}$ INSA de Rennes, CNRS, ISCR (Institut des Sciences Chimiques de Rennes) - UMR 6226, Rennes F-35000, France

*tianyingcjlu@163.com (Y.Tian),sxucjlu@163.com (S.Xu)

Abstract: Fluorescence of negative-charged nitrogen-vacancy centers in nanodiamond allow applications in quantum metrology, nanoscale sensor and bioimaging, of utmost relevance to domains from nanotechnologies to biosensing. However, the low color center content and collection efficiency of photons are crucial issues. Although, several studies about coupling nanodiamond into optical This article has been accepted for publication and undergone full peer review but has not been through the copyediting, typesetting, pagination and proofreading process, which may lead to differences between this version and the Version of Record. Please cite this article as doi: $\underline{10.1111 / \text { jace. } 17569}$ 
waveguides have already been proposed, the search for the most appropriate substance and simplest, most effective method are of keys. In this paper, we present a novel non-traditional way to incorporate nanodiamond with increased negative-charged nitrogen-vacancy content to $75.48 \%$ into tellurite germanate glass. The hybrid glass is stable in structure with predominant $\mathrm{TeO}_{4}$ trigonal bipyramid. Moreover, the fluorescence intensity is enhanced in composite. Such robust nanodiamond in tellurite germanate glass optical system has the potential to be used for nanoparticle sensing and quantum metrology.

Keywords: nanodiamond, tellurite, photoluminescence, nitrogen-vacancy, composite material

\section{1 | Introduction}

Fluorescent nanodiamond (ND) has undeniable promise in fields such as quantum key distribution, ${ }^{1}$ quantum metrology, ${ }^{2}$ nanoscale electric, magnetic and temperature field sensing, ${ }^{3-5}$ and bioimaging 6 due to its small size, great flexibility and excellent biological compatibility. Arguably, there are varieties of color centers in ND, while the negatively charged nitrogen vacancy $\left(\mathrm{NV}^{-}\right)$color centers are the most important owing to their high-brightness, stable luminescence, relatively controllable fabrication, dull bleaching and shimmering, and room-temperature spin polarization. ${ }^{7}$ As a result, most of the applications mentioned above rely on the photoluminescence of $\mathrm{NV}^{-}$color center. Whilst fluorescent nanodiamond has broad application prospects, especially in the field of quantum, two real difficulties in applying it to practical technologies are described below.

First, as the size of diamond particles reduces to the nanometer scale, only about $1 \%$ of nanodiamond contains the nitrogen vacancy (NV) center, which restricts their applications. ${ }^{8,9}$ Therefore, the artificial generation of NV centers in nanodiamond is an important challenge for various applications. So far, three approaches have applied to generate the NV centers in commercially available diamond. One is to efficiently generate NV centers by irradiating diamond with high-energy particles (electrons, protons, neutrons, etc.) to create vacancies, and then performing high temperature annealing in vacuum for the vacancies migrating towards nitrogen impurities. ${ }^{10,11}$ This most commonly used method is suitable for diamonds containing enough nitrogen impurities inside. However, this method has disadvantage of high equipment costs, time-consuming operation and some undesired results, such as electron spin resonance shift and linewidth broadening, induced 
by the high energy irradiation procedure. ${ }^{12}$ The second method generates more nitrogen substitution by implanting high-energy nitrogen ions, followed by high-temperature annealing to produce NV centers in diamonds containing deficient nitrogen impurities. ${ }^{13}$ The third approach is that the NV centers are formed by using the nitrogen impurity defect of ND itself to bind the moving vacancy through high temperature annealing in vacuum and then oxidation in air to remove the $\mathrm{sp}^{2}$-bonded carbon on the surface of ND. The concentration of NV color centers produced by this method is affected by N impurities in ND. ${ }^{14}$ Compared with the first two methods, this way has simple instrument, short operation time and low cost. In this work, therefore, NV centers were produced by the high temperature annealing method. Moreover, the ND is highly sensitive to external magnetic, electric, and temperature fields, of which the sensitivity to temperature fields is used for biological thermal measurement. ${ }^{15}$

Second, the collection efficiency of emitted photons which is extremely significant to varieties of applications is low. The collection efficiency of conventional detection approaches is only $2 \%,{ }^{16}$ When the emission of the $\mathrm{NV}^{-}$centers in bulk diamond is imaged by conventional confocal microscopy, the fluorescence collection efficiency is universally less than $10 \% .{ }^{17}$ To overcome this issue, researchers have made numerous attempts, finding that coupling the NV fluorescence emission photons into optical waveguides is an attractive solution. ${ }^{18}$ Several approaches have been explored, such as the pure diamond waveguides, ${ }^{19}$ and NDs grown on the end face of fiber, or NDs manipulated on to the end face or tapered regions to form waveguide/fiber structures. ${ }^{20-23}$ The hybrid structures produced by these methods all require sophisticated operations to attach or grow ND onto the optical fiber surface, which is too complex and inconvenient for experimental operation. In addition, NDs are on the surface of the fiber, leading to mechanical instability which restricts the applicability and life of devices. ${ }^{24}$

Thus, a method of combining NV color center quantum emitters with photonic structures to embed NDs directly into glass has attracted the attention of researchers. ${ }^{25,} 26$ However, to obtain ND/glass composite with excellent performance, it is necessary to select and design the glass system that matches the performance of ND. Based on the previous researches, ${ }^{27-29}$ it's proposed that the glass materials with ND should have the following characteristics: (1) low melting temperature and glass transition temperature $<450^{\circ} \mathrm{C}$, which minimizes the oxidation of the ND; (2) high transmittance in the NV color center excitation and emission wavelength range of 500-800nm; (3) a refractive index close to ND (2.43); (4) good chemical stability; (5) excellent anti-crystallization properties, $\Delta \mathrm{T}>100^{\circ} \mathrm{C}$, which is beneficial for drawing into fiber; (6) low content 
of hydroxyl impurities, which is easy to obtain high optical quality. In many mature glass systems, tellurite glass basically meets the above requirements. ${ }^{30-33}$ However, due to its unique triangular double-cone structure, tellurite glass has poor thermal stability and narrow fiber drawing temperature range. Moreover, high-valence state tellurium is apt to undergo redox reaction with NV centers, resulting in fluorescence extinction. ${ }^{34}$ Thus, here, a moderate amount of germanium oxide was added to the $\mathrm{TeO}_{2}-\mathrm{ZnO}-\mathrm{Na}_{2} \mathrm{O}$ glass system superseding a part of tellurium oxide to improve its thermal stability. Furthermore, boron oxide joined in the system to boost fluidity while mixing the NDs into the glass melt. ${ }^{35}$ It is worth noting that NDs will be oxidized at high temperature. ${ }^{36}$ That is the reason why NDs cannot be mixed into glass by conventional eutectic method. We used a two-step process to generate this composite: melting the raw glass material at high temperature and adding NDs at low temperature.

In this paper, we fabricated $\mathrm{NV}^{-}$center with high temperature annealing method and synthesis $\mathrm{ND} /$ tellurite germanate glass by a two-step process. The structure and luminescence of the samples were characterized by differential thermal analysis (DTA), Raman, X-ray photoelectron spectroscopy (XPS) and photoluminescence $(\mathrm{PL})$. We determined the increase of $\mathrm{NV}^{-}$color center content after high temperature annealing of NDs, and proved the successful incorporation of NDs into glass from the structure and photoluminescence point of view.

\section{2 | Experiment}

\section{1 | Fabrication of $\mathrm{NV}^{-}$centers in NDs}

The $\mathrm{NV}^{-}$centers in NDs was prepared by high temperature annealing. The ND powder used in experiment was commercially available with $60-100 \mathrm{~nm}$. The flow chart is displayed in the top section of Fig. 1. First, NDs were annealed in vacuum carbon tube furnace at $800^{\circ} \mathrm{C}$ for $2 \mathrm{~h}$ with the heating rate of $200^{\circ} \mathrm{C} / \mathrm{h}$. In the annealing process, the vacancy existing in NDs moved to the $\mathrm{N}$ substitution bit and was bounded by $\mathrm{N}$ atom to form $\mathrm{NV}^{-}$color center. The $\mathrm{sp}^{2}$ hybrid graphite phases were also produced on the surface of nanocrystals at annealing process which can inhibit $\mathrm{NV}^{-}$color center luminescence. Notably, the $400^{\circ} \mathrm{C}$ oxidation temperature of graphite is lower than $670^{\circ} \mathrm{C}$ oxidation temperature of ND. ${ }^{37}$ Therefore, it is effective to oxidize the annealed NDs in air at $550^{\circ} \mathrm{C}$ for $2 \mathrm{~h}$ to remove the $\mathrm{sp}^{2}$-bonded carbon atoms. The ND experienced the annealing and oxidation was named as treated-ND (t-ND).

\section{2 | Fabrication of glass}


The t-ND doped tellurite germanate glass with composition (in mol\%) $65 \mathrm{TeO}_{2}-15 \mathrm{ZnO}-10 \mathrm{Na}_{2} \mathrm{O}-5 \mathrm{GeO}_{2}-5 \mathrm{~B}_{2} \mathrm{O}_{3}$ was prepared by a two-step process as named tND-TZNGB. While the t-ND free tellurite germanate glass and $70 \mathrm{TeO}_{2}-20 \mathrm{ZnO}-10 \mathrm{Na}_{2} \mathrm{O}$ tellurite glass were fabricated by the same process, named as TZNGB and TZN respectively. The process is shown in the lower part of Fig. 1. Firstly, homogeneously mixed $\mathrm{TeO}_{2}, \mathrm{ZnO}$, $\mathrm{Na}_{2} \mathrm{CO}_{3}, \mathrm{GeO}_{2}$ and $\mathrm{H}_{3} \mathrm{BO}_{3}$ were melted using corundum crucible in furnace at $850^{\circ} \mathrm{C}$ for 15 minutes. Secondly, in order to prevent the t-NDs from being oxidized by high temperature during the incorporation process, temperature was reduced to $550^{\circ} \mathrm{C}$ and $0.9 \pm 0.1 \mathrm{mg}(90 \pm 10 \mathrm{ppm}) \mathrm{t}-\mathrm{NDs}$ were added into the $10 \mathrm{~g}$ melt, and kept for approximately 5-10 minutes. The t-NDs doping concentration was yielded in ppm-weight by measuring the difference between the t-NDs weight before adding and the remained amount. Even though the temperature has dropped to $550^{\circ} \mathrm{C}$, oxidation of t-NDs in the glass melt is still inevitably observed to a greater or lesser extent. If the t-NDs concentration was too small, almost all t-NDs doped into the melt might be destroyed by oxidation. At large concentration $(>100 \mathrm{ppm})$ in small glass melt $(10 \mathrm{~g})$ with reduced oxygen atmosphere, as the doping concentration rising, the reduced tellurium species increase, resulting in dark color and lower transmittance in visible. ${ }^{28}$ Therefore, we doped 90ppm t-NDs into melt, and remained the oxygen-rich atmosphere without lid. Finally, the t-ND doped melt was cast into a preheated brass mould, and annealed at $300^{\circ} \mathrm{C}\left(\sim 10^{\circ} \mathrm{C}\right.$ lower than the glass transition temperature $\left.\mathrm{T}_{\mathrm{g}}\right)$ for $4 \mathrm{~h}$ to eliminate internal stress.

\section{3 | Performance characterization}

In this experiment, the DTA of the TZNGB glass was carried out on a differential thermal analyzer (DTA404PC model, Germany) at a heating rate of $10 \mathrm{~K} / \mathrm{min}$ with alumina as a reference. Raman and PL spectra were measured by Renishaw Raman laser spectrometer using the 532nm laser of an argon ion laser as the excitation source. The focused beam was illuminated onto the sample by a L50× objective of a Leica DMLP microscope. The backscattered signals from sample were collected through the same objective and detected by a CCD array detector. Chemical bonding states in glass was examined using XPS. The XPS used a monochromatic $\mathrm{Al} \mathrm{K \alpha}(1486.6 \mathrm{eV})$ as the source. For the initial survey spectra and the high-resolution spectra, pass energy of $150 \mathrm{eV}$ with the step size of $1.0 \mathrm{eV}$ and $30 \mathrm{eV}$ with a step size of $0.05 \mathrm{eV}$ were applied, respectively. Te $3 \mathrm{~d}$ peak at $576 \mathrm{eV}$ was performed as reference to energy calibration the binding energies (BE) of photoelectron spectra. The processing of the measured spectra includes a subtraction of X-ray satellites and a mixed of Gaussian-Lorentzian function for the XPS peak fitting. 


\section{3 | Results and Discussions}

The DTA of TZN and TZNGB glass were tested with heating rate of $10^{\circ} \mathrm{C} / \mathrm{min}$ at room temperature, illustrated as Fig. 2(a). Besides, the parameters about differential thermal of TZN and TZNGB glass were signed in graph. The glass transition temperature $\left(\mathrm{T}_{\mathrm{g}}\right)$ lies around $314^{\circ} \mathrm{C}$, which is slightly higher than that of traditional $\mathrm{TeO}_{2^{-}}$ $\mathrm{ZnO}-\mathrm{Na}_{2} \mathrm{O}(\mathrm{TZN})$ system glass $\left(303^{\circ} \mathrm{C}\right)$. Meanwhile the temperature of $447^{\circ} \mathrm{C}$ corresponds to the crystallization onset temperature $\left(\mathrm{T}_{\mathrm{x}}\right)$ of TZNGB glass. $\triangle \mathrm{T}$ has been frequently used as a rough measure of the

glass thermal stability, which is identified as: $\Delta \mathrm{T}=\mathrm{T}_{\mathrm{x}}-\mathrm{T}_{\mathrm{g}} \cdot{ }^{38} \mathrm{~A}$ large $\Delta \mathrm{T}$ means strong inhibition of nucleation and crystallization during the fiber fabricating, reducing the scattering loss. Compare with TZN glass, the large $\Delta \mathrm{T}$ of TZNGB glass $\left(133^{\circ} \mathrm{C}\right)$ in this experiment suggests that the introducing $\mathrm{B}_{2} \mathrm{O}_{3}$ and $\mathrm{GeO}_{2}$ into tellurite glass improved their thermal stability. It might be due to the replacement of part $\mathrm{TeO}_{2}$ by $\mathrm{B}_{2} \mathrm{O}_{3}$ and $\mathrm{GeO}_{2}$ changing the structure of glass, which will be demonstrated by the subsequent Raman test. Moreover, the transmittance at 500-800nm is excellent, shown in Fig. 2(b). The lower transmittance of tND-TZNGB glass than TZNGB glass can be explained by the illustration. It is obvious that a slight coloration exists in tNDTZNGB glass caused by t-ND incorporation, producing scattering and reducing transmittance. Even though the transmittance has decreased, it still has a high transmittance in the NV color center excitation and emission wavelength range of $500-800 \mathrm{~nm}$. The $\mathrm{TeO}_{2}-\mathrm{ZnO}-\mathrm{Na}_{2} \mathrm{O}-\mathrm{GeO}_{2}-\mathrm{B}_{2} \mathrm{O}_{3}$ glass is suitable for nanodiamond composite.

Raman is one of the most frequently used characterization methods to analyze glass internal network structure. In order to study the performance of tellurite glass with introduction of $\mathrm{GeO}_{2}$ and $\mathrm{B}_{2} \mathrm{O}_{3}$, normalized Raman spectra of TZN and TZNGB glass were carried out and described at Fig. 3 in detail. The spectrum is characterized by three polarized bands corresponding to a broad band near $453 \mathrm{~cm}^{-1}$, a shoulder band around $678 \mathrm{~cm}^{-1}$, and another band about $756 \mathrm{~cm}^{-1}$. The above bands have been contributed by three different types of stretching vibrations, i.e. , the stretching modes of Te-O-Te or O-Te-O linkage, stretching modes of Te-O bond in $\mathrm{TeO}_{4}$ trigonal bipyramid (tbp), and bending modes of $\mathrm{Te}-\mathrm{O}$ bond in $\mathrm{TeO}_{3+1}$ or $\mathrm{TeO}_{3}$ trigonal pyramid (tp) units, respectively. These characteristic peaks are similar to those reported previously for tellurite glasses. ${ }^{39-41}$ Despite the shape of the bands are not changed by bits of $\mathrm{GeO}_{2}$ and $\mathrm{B}_{2} \mathrm{O}_{3}$ addition, slight shifts of band positions happen in TZNGB glass. The blue shift of Te-O-Te stretching vibration is due to the deformation of 
Te-O-Te bond caused by the introduction of $\mathrm{GeO}_{2} \cdot{ }^{42-44}$ The blue shift at near $678 \mathrm{~cm}^{-1}$ might be attributed to the formation of $\left[\mathrm{GeO}_{4}\right]^{4}$ units after the addition of $\mathrm{GeO}_{2}$, which can provide partial oxygen ions to achieve the network continuity and relatively stability in a tellurite glass structure. ${ }^{45-47}$

Raman and PL measurement of t-ND that have treated by annealing and oxidation and raw ND powder were performed, as shown in Fig. 4 and 5 respectively. In Fig. 4, the Raman characteristic peak of diamond appears at $1332 \mathrm{~cm}^{-1}$. The higher intensity $\mathrm{sp}^{3}$ diamond peak in t-ND indicates high temperature annealing process is useful. An obvious band near $1580 \mathrm{~cm}^{-1}$ in raw ND corresponds to the graphite Raman feature (G peak), while t-ND exhibits a small one. It represents that graphitization came up during the annealing process, following that the oxidation process eliminating the graphite.

Figure 5 (a) and (b) exhibit the fluorescence spectra of t-ND and ND measured under 532nm excitation at $80 \mathrm{~K}$ and $300 \mathrm{~K}$ temperature, respectively. In order to ensure the comparability, they were obtained at the same pump power of $5 \mathrm{~mW}$, uniform integral time of $1 \mathrm{~s}$ and identical integral number of one time per second. PL spectra show that both raw ND powder and t-ND perform $\mathrm{NV}^{-}$and $\mathrm{NV}^{0}$ (the neutrally charged nitrogenvacancy color center) zero-phonon line (ZPL) luminescence peaks at $575 \mathrm{~nm}$ and $638 \mathrm{~nm}$ separately, regardless of at $80 \mathrm{~K}$ or $300 \mathrm{~K}$, which is marked in Fig. 6 (a) and (b). As show in Fig. 6 (c), NV- ZPL of t-ND at 80K can be fitted by a Lorentzian function, along with an exponential baseline: 48

$$
\mathrm{I}=y_{0}+\operatorname{aexp}\left(-\frac{\lambda}{t}\right)+\frac{2 A}{\pi} \cdot \frac{\omega}{4\left(\lambda-\lambda_{0}\right)^{2}+\omega^{2}}
$$

Where $\mathrm{I}$ is the fluorescence intensity, $\lambda$ is wavelength, $\omega$ is the Lorentzian half-width, $A$ is the Lorentzian area, $\lambda_{0}$ is the ZPL center, and $y_{0}, a$, and $t$ are fitting parameters. From this formula, the integral area $A$ of NV- ZPL was obtained, the pink region in Fig. 6 (c). The baseline was subtracted to remove the influence of phonon sideband through this fitting program. The integral area $A$ of $\mathrm{NV}^{-} \mathrm{ZPL}$ and $\mathrm{NV}^{0} \mathrm{ZPL}$ of ND and t-ND at 80K were obtained by the same procedure. The $\mathrm{NV}^{-0} \%\left(N^{-} \%=\frac{A_{N V^{-}}}{A_{N V^{-}}+A_{N V^{0}}} \times 100 \%\right)$ of the t-ND and ND at $80 \mathrm{~K}$ is $75.48 \%$ and $59.82 \%$, respectively. The enlargement of $\mathrm{NV}^{-}$content confirms that the $\mathrm{NV}^{-}$color center was efficiently formed by high temperature annealing. Meanwhile, the $\mathrm{NV}^{-} \mathrm{ZPL}$ integral area of t-ND at 80K is five times of that at $300 \mathrm{~K}$, indicating the promotion of low temperature in $\mathrm{NV}^{-}$center emitting. This difference demonstrates the temperature dependence of fluorescence.

As the addition of t-ND powder into the TZNGB glass, from the Raman curves in Fig. 6(a), the $\mathrm{sp}^{3}$ diamond characteristic peak at $1332 \mathrm{~cm}^{-1}$ and three Raman bands representing tellurite glass at $455 \mathrm{~cm}^{-1}, 682 \mathrm{~cm}^{-}$ 
${ }^{1}$ and $756 \mathrm{~cm}^{-1}$ are observed. Thus, the t-ND powders were successfully doped in TZNGB glass. The upward trend of the baseline at $1000-2000 \mathrm{~cm}^{-1}$ could be due to the influence of t-ND fluorescence. In addition to ND and t-ND, the PL spectra of t-ND doped glass was also measured at 80K with 532nm laser, displayed in Fig. 6(b). Markedly, $\mathrm{NV}^{0} \mathrm{ZPL}, \mathrm{NV}^{-} \mathrm{ZPL}$ and $\mathrm{NV}^{-}$phonon sideband exist, that is similar to PL spectra of t-ND. Comparing to tND-TZNGB glass, peak in PL curve of TZNGB glass is absent and there is no luminescence at $560-800 \mathrm{~nm}$. It is confirmed again the presence of t-ND and the coupling of NV centers in tND-TZNGB glass. The peaks at $552 \mathrm{~nm}$ and $554 \mathrm{~nm}$ are corresponding to TZNGB glass Raman bands at $682 \mathrm{~cm}^{-1}$ and $756 \mathrm{~cm}^{-1}$. Calculating the ratio of $\mathrm{NV}^{-}$to $\mathrm{NV}^{0}$ integration peaks in the PL spectra, the value of $\mathrm{NV}^{-}$relative to total $\mathrm{NV}$ in TZNGB glass is 1.4 higher than t-ND. Besides, the fluorescence intensity of $\mathrm{NV}^{-} \mathrm{ZPL}$ in composite is stronger than that of $\mathrm{t}-\mathrm{ND}$. During the doping procedure, high temperature further removed the $\mathrm{sp}^{2}$ carbon from the surface and promoted the formation of oxygen-containing functional groups at nanodiamond surface termination. These changes occurred at nanodiamond surface are benefit of the NV- ZPL fluorescence. ${ }^{49}$ These results indicate that the $\mathrm{NV}^{-}$properties are stable in prepared tND-TZNGB glass.

XPS measurement was performed to elucidate the chemical state and local chemical environment of elements in the tND-TZNGB glass, including survey scan and high resolution scan, as shown in Fig. 7. From the XPS survey scan (Fig. 7(a)), peaks corresponding to tellurium, oxygen, boron, zinc, germanium, sodium and carbon were observed. The presence of these elements in the glass substantiates that the previously designed components have been successfully introduced into the glass. High-resolution scan spectra of Te3d, O1s and C1s of $\mathrm{tND}-\mathrm{TZNGB}$ glass were undertaken to determine the elemental composition.

Figure 7(b) presents the high-resolution scan and the fitted peak for Te3d spectra. The peaks at $586.4 \mathrm{eV}$ and $576.3 \mathrm{eV}$ respectively corresponding to the doublets $T e 3 \mathrm{~d}_{3 / 2}$ and $T e 3 \mathrm{~d}_{5 / 2}$ were observed, according with the report for amorphous $\mathrm{TeO}_{2}{ }^{50}$ The $\mathrm{Te} 3 \mathrm{~d}_{5 / 2}$ peak of the examined glass is deconvoluted into two peaks contributed at 575.0 and $576.3 \mathrm{eV}$. According to prior studies, ${ }^{51,52}$ these binding energy (BE) are related to the presence of $\mathrm{TeO}_{3}$ trigonal pyramid (tp) and $\mathrm{TeO}_{4}$ trigonal bipyramid (tbp) units, respectively. The structure stick model of $\mathrm{TeO}_{3}$ tp and $\mathrm{TeO}_{4}$ tbp were given in illustration (1) and (2), respectively. The peak positions and fitting details for Te3 $\mathrm{d}_{5 / 2}$ orbital including FWHM and area percentage are listed in Table 1. The concentration of the substance can be represented by the fitting peak area. Obviously, the $88.46 \%$ area percentage of $\mathrm{TeO}_{4}$ tbp is higher than $11.54 \%$ of $\mathrm{TeO}_{3}$ tp. Conclusively, both $\mathrm{TeO}_{4}$ and $\mathrm{TeO}_{3}$ units consist in tND-TZNGB glass but the $\mathrm{TeO}_{4}$ amount is predominant in the amorphous structure, which is in agreement with previous XPS 
studies of alkali tellurite glasses. ${ }^{44,} 52,53$ The alkali metal oxide $\mathrm{Na}_{2} \mathrm{O}$ converts $\mathrm{TeO}_{4}$ tbp to $\mathrm{TeO}_{3}$ tbp by disrupting the Te-O-Te bonds in the network structure, which affects the coherence and thermal stability of the network structure. The incorporation of cations with charge greater than one, such as $\mathrm{B}^{3+}$ and $\mathrm{Ge}^{4+}$, leads connection of lone pair electrons on tbp and tp, which enhances the continuity of the network structure and improves the thermal stability. The schematic was displayed in illustration (3). Besides, the addition of nanodiamond did not change the structure of TZNGB glass matrix.

In O1s high resolution spectrum (Fig. 7(c)), a slight asymmetric peak at $530.8 \mathrm{eV}$ can be fitted to three peaks corresponding to the peak center of $529.9 \mathrm{eV}, 530.8 \mathrm{eV}$ and $532.2 \mathrm{eV}$. It might be indicative of contributions from different oxygen sites: NBO (nonbridging oxygen, oxygen links a glass former and a glass modifier or double bonds to a glass former), BO (bridging oxygen, an oxygen links two glass former in the glass network), and Te-O-Ge or (and) Te-O-B bonds. The O1s lines for NBO and BO are known to be about 529-530 eV and 531-532 eV respectively, which are affected by electronegativity and electron density. BO peak always has higher BE than NBO peak because glass former Te bonded by BO has larger electronegativity compared to glass modifiers $\mathrm{Zn}$ and Na. Electrons are apt to be attracted by high electronegative elements when elements form bonds with $\mathrm{O}$. Then the reduction of electron density around $\mathrm{BO}$ leads to $\mathrm{BE}$ of core electrons increasing. ${ }^{53}$ According to XPS analysis, we conclude that the NBO at $529.9 \mathrm{eV}$ is assigned to Te-O-Na and $\mathrm{Te}=\mathrm{O}$, while the $\mathrm{BO}$ at $530.7 \mathrm{eV}$ is distributed to Te-O-Te. In general, O1s core-level spectrum is deconvoluted to NBO and BO peaks. But, in order to better match the measurement curve, the third peak was fitted. C.L. Medrano-Pesqueira et al studied the structure of $\mathrm{CdO}-\mathrm{TeO}_{2}-\mathrm{GeO}_{2}$ system glass, and attributed the fitting peak at $531.64 \mathrm{eV}$ of O1s core-level to $\mathrm{GeO}_{2} \cdot{ }^{51} \mathrm{In} \mathrm{TeO}_{2}-\mathrm{PbO}-\mathrm{TiO}_{2}-\mathrm{B}_{2} \mathrm{O}_{3}$ system glass reported by $\mathrm{Chen}$, $\mathrm{B}-\mathrm{O}$ bond was responsible for the fitted peak at $533 \mathrm{eV} .{ }^{54}$ Based on these results, we suggest that the peak at $532.0 \mathrm{eV}$ should belong to Te-O-Ge or Te-O-B bond or both. In combination of Raman spectra, it is concluded that the structure of this glass sample is formed by predominant $\mathrm{TeO}_{4}$ tbp with several $\mathrm{BOs}$, a small amount of $\mathrm{TeO}_{3}$ tp with two BOs and one NBO, and little Te-O-Ge or Te-O-B bonds, as shown as the illustration in Fig. 7(c).

The C1s core level XPS spectra of the tND-TZNGB glass are shown as Fig. 7(d). The C1s peak demonstrates the presence of several components, including $\mathrm{sp}^{2}$-hybridised carbon, $\mathrm{sp}^{3}$-hybridized carbon species, and oxygen-bonded carbon components. A sharp peak centered at $285.2 \mathrm{eV}$ can be attributed to $\mathrm{sp}^{3}$ hybridised carbon species, which is the feature structure of diamond. The diamond structure was pictured in illustration (2) of Fig. 7(d). The C1s line in graphite occurs at lower binding energy than that in diamond, ${ }^{55}$ so 
the peak at $284.4 \mathrm{eV}$ is assigned to $\mathrm{sp}^{2}$ carbon. The $\mathrm{C}=\mathrm{C}$ bond structure model was drawn in illustration (1) of Fig. 7(d). Another peak at $285.9 \mathrm{eV}$ may be contributed by $\mathrm{C}-\mathrm{O}$ bond as it is similar with results of Guillevic Mazheva et al. ${ }^{56}$ It may be caused by residual $\mathrm{Na}_{2} \mathrm{CO}_{3}$ materials after decomposition or a small amount of nanodiamond oxidation. The fitted curve of $\mathrm{sp}^{3}$-hybridized carbon species has the highest intensity signifying that the $\mathrm{C}$ element in the testing range is principally $\mathrm{sp}^{3}$ hybrid diamond structure. It substantiates the existence of t-ND in glass network.

\section{4 | Conclusion}

Nanodiamonds experienced high temperature vacuum annealing and oxidation to synthesize more $\mathrm{NV}^{-}$colour centers. This technology is feasible compared with conventional approaches of highenergy electron irradiation. We observed the increase of $\mathrm{NV}^{-}$content from $59.82 \%$ to $75.48 \%$ and the enhanced fluorescence intensity.

The t-ND doped TZNGB glass was prepared by a two-step process, which directly coupled the t-ND to TZNGB glass. It overcomes the disadvantages from method of evanescent coupling diamond with photonic devices. The Raman and PL spectra confirmed the presence of nanodiamonds in TZNGB glass. The fluorescence curves show that the $\mathrm{NV}^{-}$properties are stable in prepared tND-TZNGB glass. From Raman spectra and XPS, we obtained that the structure of $\mathrm{tND}$-TZNGB is formed by predominant $\mathrm{TeO}_{4}$ tbp, a small amount of $\mathrm{TeO}_{3} \mathrm{tp}$, and little Te-O-Ge or Te-O-B bonds. The structure was hardly affected by t-ND doping. And this hybrid material combined with optical waveguide and quantum emission is a promising candidate of nano-sensing and quantum metrology.

\section{Funding}

This work was supported by the National Natural Science Foundation of China (NSFC) (No. 61775205, 51872270, 61975193, 51702307), the Fundamental Research Funds for the Provincial Universities of Zhejiang and Science and Technology Innovation Platform and Talent Plan of Zhejiang. 


\section{References}

1. Hassanzadeh P. Towards the quantum-enabled technologies for development of drugs or delivery systems. J Control Release. 2020; 324: 260-79.

2. Schmunk W, Rodenberger M, Peters S, Hofer H, Kuck S. Radiometric calibration of single photon detectors by a single photon source based on NV- centers in diamond. J Mod Optic. 2011; 58(14): 1252-9.

3. Dolde F, Fedder H, Doherty MW, Nobauer T, Rempp F, Balasubramanian G, et al. Electric-field sensing using single diamond spins. Nature Physics. 2011; 7(6):459-63.

4. Kucsko G, Maurer PC, Yao NY, Kubo M, Noh HJ, Lo PK, et al. Nanometre-scale thermometry in a living cell. Nature. 2013; 500: 54.

5. Balasubramanian G, Chan IY, Kolesov R, Al-Hmoud M, Tisler J, Shin C, et al. Nanoscale imaging magnetometry with diamond spins under ambient conditions. Nature. 2008; 455: 648.

6. Torelli MD, Nunn NA, Shenderova OA. A Perspective on Fluorescent Nanodiamond Bioimaging. Small. 2019; 15(48): 20.

7. Wang KY, Steeds JW, Li ZH, Tian YM. Photoluminescence Studies of Both the Neutral and Negatively Charged Nitrogen-Vacancy Center in Diamond. Microsc Microanal. 2016; 22(1): 108-12.

8. Bradac C, Gao WB, Forneris J, Trusheim ME, Aharonovich I. Quantum nanophotonics with group IV defects in diamond. Nat Commun. 2019; 10: 13.

9. Nguyen M, Nikolay N, Bradac C, Kianinia M, Ekimov EA, Mendelson N, et al. Photodynamics and quantum efficiency of germanium vacancy color centers in diamond. Adv Photonics. 2019; 1(6).

10. Botsoa J, Sauvage T, Adam MP, Desgardin P, Leoni E, Courtois B, et al. Optimal conditions for $\mathrm{NV}^{-}$center formation in type-1b diamond studied using photoluminescence and positron annihilation spectroscopies. Phys Rev B. 2011; 84(12).

11. Ashfold MNR, Goss JP, Green BL, May PW, Newton ME, Peaker CV. Nitrogen in Diamond. Chem Rev. 2020; 120(12): 5745-94.

12. Kim E, Acosta VM, Bauch E, Budker D, Hemmer PR. Electron spin resonance shift and linewidth broadening of nitrogen-vacancy centers in diamond as a function of electron irradiation dose. Appl Phys Lett. 2012; 101(8).

13. Pezzagna S, Naydenov B, Jelezko F, Wrachtrup J, Meijer J. Creation efficiency of nitrogen-vacancy centres in diamond. New J Phys. 2010; 12.

14. Song X, Wang G, Liu X, Feng F, Wang J, Lou L, et al. Generation of nitrogen-vacancy color center in nanodiamonds by high temperature annealing. Appl Phys Lett. 2013; 102(13): 133109.

15. Tsai PC, Epperla CP, Huang JS, Chen OY, Wu CC, Chang HC. Measuring Nanoscale Thermostability of Cell Membranes with Single Gold-Diamond Nanohybrids. Angewandte Chemie. 2017; 56(11): 3025-30. 
16. Taylor JM, Cappellaro P, Childress L, Jiang L, Budker D, Hemmer PR, et al. High-sensitivity diamond magnetometer with nanoscale resolution. Nature Physics. 2008; 4(10): 810-6.

17. Castelletto S, Harrison JP, Marseglia L, Stanley-Clarke AC, Gibson BC, Fairchild BA, et al. Diamond-based structures to collect and guide light. New J Phys. 2011; 13: 025020.

18. Jia Y, Chen F. Compact solid-state waveguide lasers operating in the pulsed regime: a review Invited. Chinese Opt Lett. 2019; 17(1).

19. Lopez-Lorente AI, Wang P, Sieger M, Catalan EV, Karlsson M, Nikolajeff F, et al. Mid-infrared thin-film diamond waveguides combined with tunable quantum cascade lasers for analyzing the secondary structure of proteins. Phys Status Solidi A. 2016; 213(8): 2117-23.

20. Dory C, Vercruysse D, Yang KY, Sapra NV, Rugar AE, Sun S, et al. Inverse-designed diamond photonics. Nat Commun. 2019; 10: 7.

21. Babinec TM, Hausmann BJM, Khan M, Zhang YA, Maze JR, Hemmer PR, et al. A diamond nanowire singlephoton source. Nat Nanotechnol. 2010; 5(3): 195-9.

22. Rodulaski M, Zhang J, Tzeng YK, Lagoudakis KG, Ishiwata H, Dory C, et al. Nanodiamond Integration with Photonic Devices. Laser Photon Rev. 2019; 13(8): 14.

23. Ampem-Lassen E, Simpson DA, Gibson BC, Trpkovski S, Hossain FM, Huntington ST, et al. Nanomanipulation of diamond-based single photon sources. Opt Express. 2009; 17(14): 11287-93.

24. Ruan Y, Simpson D, Jeske J, Ebendorff-Heidepriem H, Lau D, Ji H, et al. Magnetically sensitive nanodiamonddoped tellurite glass fibers. Sci Rep. 2018; 8.

25. Yang Z, Mei Y, Chen C, Ruan Y, Hu X. Synthesis of strong SiV photoluminescent diamond particles on silica optical fiber by chemical vapor deposition. Chinese Phys B. 2018; 27(3): 038101.

26. Ruan Y, Gibson BC, Lau DW, Greentree AD, Ji H, Ebendorff-Heidepriem H, et al. Atom-Photon Coupling from Nitrogen-vacancy Centres Embedded in Tellurite Microspheres. Sci Rep. 2015; 5: 11486.

27. Henderson MR, Gibson BC, Ebendorff-Heidepriem H, Kuan K, Afshar VS, Orwa JO, et al. Diamond in tellurite glass: a new medium for quantum information. Adv Mater. 2011; 23(25): 2806-10.

28. Ebendorff-Heidepriem H, Ruan Y, Ji H, Greentree AD, Gibson BC, Monro TM. Nanodiamond in tellurite glass Part I: origin of loss in nanodiamond-doped glass. Opt Mater Express. 2014; 4(12): 2608.

29. Qiang B, Dubrovkin AM, Krishnamoorthy HNS, Wang Q, Soci C, Zhang Y, et al. High Q-factor controllable phononic modes in hybrid phononic-dielectric structures. Adv Photonics. 2019; 1(2).

30. Wang L, Cao J, Lu Y, Li X, Xu S, Zhang Q, et al. In situ instant generation of an ultrabroadband near-infrared emission center in bismuth-doped borosilicate glasses via a femtosecond laser. Photonics Res. 2019; 7(3): 30010. 
31. Zheng QY, Li Y, Wu WJ, Zou J, Yang BB, Shi MM. Effect of reduction co-sintering on the photoluminescence properties of phosphor in glass in boro-bismuthate glass. Chinese Opt Lett. 2019; 17(10): 5.

32. Yao T, Huang L, Zhou P, Lei B, Leng J, Chen J. Power scaling on tellurite glass Raman fibre lasers for midinfrared applications. High Power Laser Sci Eng. 2018; 6.

33. Huault M, Luis DD, Apianiz JI, Marco MD, Salgado C, Gordillo N, et al. A 2D scintillator-based proton detector for high repetition rate experiments. High Power Laser Sci Eng. 2019; 7(4).

34. Ruan Y, Ji H, Johnson BC, Ohshima T, Greentree AD, Gibson BC, et al. Nanodiamond in tellurite glass Part II: practical nanodiamond-doped fibers. Opt Mater Express. 2014; 5(1): 73.

35. Li TT, Segawa H, Ohashi N. Sintering behavior and dielectric properties of BaTiO3 added with BaO-Bi2O3B2O3 glass phase. Ceram Int. 2018; 44(11): 13004-10.

36. Gao J, Wang Y, Wu HH, Liu X, Wang LL, Yu QL, et al. Construction of a sp $3 / \mathrm{sp}^{2}$ Carbon Interface in 3D NDoped Nanocarbons for the Oxygen Reduction Reaction. Angew Chem Int Edit. 2019; 58(42): 15089-97.

37. Smith BR, Gruber D, Plakhotnik T. The effects of surface oxidation on luminescence of nano diamonds. Diam Relat Mater. 2010; 19(4): 314-8.

38. Ren F, Mei YZ, Gao C, Zhu LG, Lu AX. Thermal stability and Judd-Ofelt analysis of optical properties of $\mathrm{Er}^{3+}$-doped tellurite glasses. T Nonferr Metal Soc. 2012; 22(8): 2021-6.

39. Santos FA, Figueiredo MS, Barbano EC, Misoguti L, Lima SM, Andrade LHC, et al. Influence of lattice modifier on the nonlinear refractive index of tellurite glass. Ceram Int. 2017; 43(17): 15201-4.

40. Tang W, Tian Y, Li B, Xu Y, Liu Q, Zhang J, et al. Effect of introduction of $\mathrm{TiO}_{2}$ and $\mathrm{GeO}_{2}$ oxides on thermal stability and $2 \mu \mathrm{m}$ luminescence properties of tellurite glasses. Ceram Int. 2019; 45(13): 16411-6.

41. Photonics A. Transforming optics: an interview with Sir John Pendry. Adv Photonics. 2019; 1(1): 010502.

42. Ghribi N, Dutreilh-Colas M, Duclère JR, Gouraud F, Chotard T, Karray R, et al. Structural, mechanical and optical investigations in the $\mathrm{TeO}_{2}$-rich part of the $\mathrm{TeO}_{2}-\mathrm{GeO}_{2}-\mathrm{ZnO}$ ternary glass system. Solid State Sci. 2015; 40: 20-30.

43. Carrillo-Torres RC, Saavedra-Rodriguez G, Alvarado-Rivera J, Caldino U, Sanchez-Zeferino R, AlvarezRamos ME. Tunable emission and energy transfer in $\mathrm{TeO}_{2}-\mathrm{GeO}_{2}-\mathrm{ZnO}$ and $\mathrm{TeO}_{2}-\mathrm{GeO}_{2}-\mathrm{MgCl}_{2}$ glasses activated with $\mathrm{Eu}^{3+} / \mathrm{Dy}^{3+}$ for solid state lighting applications. J Lumin. 2019; 212: 116-25.

44. Guo JT, Wang JF, Wei H, Huang WF, Huang TR, Xia G, et al. High-power, Joule-class, temporally shaped multi-pass ring laser amplifier with two Nd: glass laser heads. High Power Laser Sci Eng. 2019; 7(01): 64-71.

45. Jha A, Richards B, Jose G, Teddy-Fernandez T, Joshi P, Jiang X, et al. Rare-earth ion doped $\mathrm{TeO}_{2}$ and $\mathrm{GeO}_{2}$ glasses as laser materials. Progress in Materials Science. 2012; 57: 1426-91.

46. Truong LN, Dussauze M, Allix M, Chenu S, Cardinal T, Fargin E, et al. Second harmonic generation in germanotellurite bulk glass-ceramics. J Am Ceram Soc. 2017; 100(4): 1412-23. 
47. Wang PF, Shao BJ, Su HP, Lv XL, Li YY, Peng YJ, et al. High-repetition-rate, high-peak-power 1450 nm laser source based on optical parametric chirped pulse amplification. High Power Laser Sci Eng. 2019; 7(02): 83-7.

48. Plakhotnik T, Aman H, Chang HC. All-optical single-nanoparticle ratiometric thermometry with a noise floor of $0.3 \mathrm{~K} \mathrm{~Hz}^{-1 / 2}$. Nanotechnology. 2015; 26(24): 245501.

49. Fu KMC, Santori C, Barclay PE, Beausoleil RG. Conversion of neutral nitrogen-vacancy centers to negatively charged nitrogen-vacancy centers through selective oxidation. Appl Phys Lett. 2010; 96(12).

50. Ogbuu O, Du Q, Lin H, Li L, Zou Y, Koontz E, et al. Impact of Stoichiometry on Structural and Optical Properties of Sputter Deposited Multicomponent Tellurite Glass Films. J Am Ceram Soc. 2015; 98(6): 1731-8.

51. Medrano-Pesqueira CL, Rodriguez-Carvajal DA. Structural properties of poly-crystals embedded in glassy matrix of the ternary system $\mathrm{CdO}-\mathrm{TeO}_{2}-\mathrm{GeO}_{2}$. J Non Cryst Solids. 2017; 475: 15-24.

52. Bachvarova-Nedelcheva A, Iordanova R, Kostov KL, Ganev V, Yordanov S. Synthesis, characterization and optical properties of non-traditional tellurite-selenite glasses. Opt Mater. 2014; 36(8): 1319-28.

53. Ahmad Kamil S, Chandrappan J, Portoles J, Steenson P, Jose G. Local structural analysis of erbium-doped tellurite modified silica glass with x-ray photoelectron spectroscopy. Mater Res Express. 2019; 6(8): 086220.

54. Chen Q, Zhang M, Wang H, Wang Q, Ma Q, Li J. Structures and magneto optical property of diamagnetic $\mathrm{TiO}_{2}-\mathrm{TeO}_{2}-\mathrm{PbO}-\mathrm{B}_{2} \mathrm{O}_{3}$ glass. J Non Cryst Solids. 2017; 468: 58-66.

55. Xie FY, Xie WG, Gong L, Zhang WH, Chen SH, Zhang QZ, et al. Surface characterization on graphitization of nanodiamond powder annealed in nitrogen ambient. Surf Interface Anal. 2010; 42(9): 1514-8.

56. Guillevic M, Pichot V, Fioux P, Schnell F, Spitzer D. Nanodiamond-based energetic core-shell composites: The route towards safer materials. Diam Relat Mater. 2019; 93: 150-8. 


\section{Table}

Table 1. Details of the Curve Fitting of Te $3 \mathrm{~d}_{5 / 2}$ Core Level Spectra.

\begin{tabular}{cccc}
\hline $\begin{array}{c}\text { Binding } \\
\text { Energy/eV } \\
( \pm 0.20)\end{array}$ & $\begin{array}{c}\text { FWHM/eV } \\
( \pm 0.20)\end{array}$ & $\begin{array}{c}\text { Area/\% } \\
( \pm 1.0)\end{array}$ & $\begin{array}{c}\text { Peak } \\
\text { Species }\end{array}$ \\
\hline 575.0 & 3.26 & 11.54 & $\mathrm{TeO}_{3}^{52}$ \\
576.3 & 1.45 & 88.46 & $\mathrm{TeO}_{4}{ }^{53}$ \\
\hline
\end{tabular}




\section{Figure captions}

Fig. 1. The flow chart of fabricating the $\mathrm{NV}^{-}$centers in NDs and t-ND doped tellurite germanate glass.

Fig. 2. (a) DTA curves of TZN and TZNGB glass with heating rate of $10^{\circ} \mathrm{C} / \mathrm{min}$ in the atmosphere. The $\mathrm{T}_{\mathrm{g}}$ and $\mathrm{T}_{\mathrm{x}}$ all marked in graph. (b) Transmittance curves of TZNGB and tND-TZNGB glass. The inset is optical photograph of TZNGB and tND-TZNGB glasses.

Fig. 3. Raman spectra of TZN and TZNGB glass in the $100-1000 \mathrm{~cm}^{-1}$ range excited by $532 \mathrm{~nm}$ at room temperature.

Fig. 4. Raman spectra in range of $300-2000 \mathrm{~cm}^{-1}$ excited by a $532 \mathrm{~nm}$ laser at room temperature. The peaks at $1332 \mathrm{~cm}^{-1}$ mean the $\mathrm{sp}^{3}$ diamond characteristic peak, and that at $1580 \mathrm{~cm}^{-1}$ is graphite $\mathrm{G}$ peak.

Fig. 5. PL spectra in range of 550-800nm spectra of raw ND powder (black solid line) and t-ND (red dotted line) excited by $532 \mathrm{~nm}$ at (a) $80 \mathrm{~K}$ and (b) $300 \mathrm{~K}$. They show the NV ${ }^{0} \mathrm{ZPL}$ at $575 \mathrm{~nm}, \mathrm{NV}^{-} \mathrm{ZPL}$ at $638 \mathrm{~nm}$ and $\mathrm{NV}^{-}$phonon sidebands at $650-800 \mathrm{~nm}$. (c) Fluorescence spectrum of t-ND at $80 \mathrm{~K}$ over $610-657 \mathrm{~nm}$ excited by $532 \mathrm{~nm}$. The red solid curve is the best-fit of the data (green curve) with exponential baseline (black curve). The pink area represents the integral area of NV-.

Fig. 6. (a) The Raman curves of tND-TZNGB and TZNGB glass; (b) PL spectra in 560-800nm of tND-TZNGB and TZNGB glass excited by 532nm at $80 \mathrm{~K}$. The tND-TZNGB glass has the sharp peaks at $573 \mathrm{~nm}, 638 \mathrm{~nm}$ and a broad peak at $650-800 \mathrm{~nm}$ owing to the luminescence of NV color center in t-ND.

Fig. 7. (a) XPS survey scan of tND-TZNGB. (b) High resolution of Te $3 d$ core-level. Te $3 d_{5 / 2}$ at $576.3 \mathrm{~nm}$ was fitted with two peaks which were assigned to $\mathrm{TeO}_{3}$ trigonal pyramid (tp) and $\mathrm{TeO}_{4}$ trigonal bipyramid (tbp). Illustration (1) and (2) is the structure model of $\mathrm{TeO}_{3}$ tp and $\mathrm{TeO}_{4}$ tbp respectively. Illustration (3) is schematic of Ge element connecting $\mathrm{TeO}_{3}$ tp and $\mathrm{TeO}_{4}$ tbp: A lone pair electrons site is like an oxide $\left(\mathrm{O}^{2-}\right)$ ion. It offers equilibrium positions through interchange via delocalization when $\left[\mathrm{GeO}_{4}\right]^{4-}$ is incorporated into the structure for achieving the network continuity and thermal stability enhancement. (c) High resolution of O1s core-level. It was fitted with three peaks corresponding to nonbridging oxygen (NBO), bridging oxygen (BO), Te-O-Ge/Te-O-B 
respectively. Illustration shows structure model of tND-TZNGB glass sample. (d) High resolution of $\mathrm{C} 1$ s core-level. It was fitted with three peaks corresponding to $\mathrm{C}=\mathrm{C}$ bond $\mathrm{sp}^{2}, \mathrm{C}-\mathrm{C}$ bond $\mathrm{sp}^{3}$ and $\mathrm{C}-\mathrm{O}$ bond. The structure model of $\mathrm{C}=\mathrm{C}$ bond and $\mathrm{C}-\mathrm{C}$ bond in diamond are shown in illustration (1) and (2) respectively. 


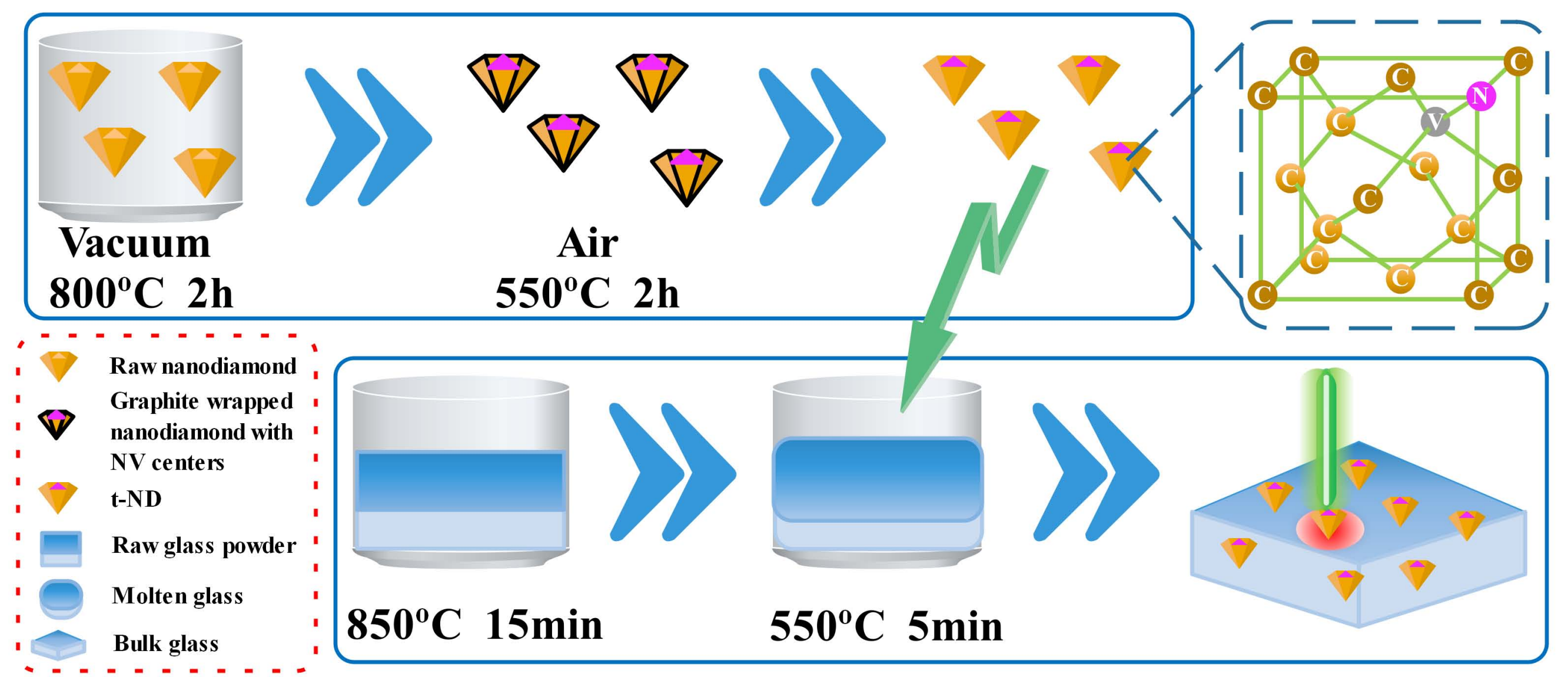




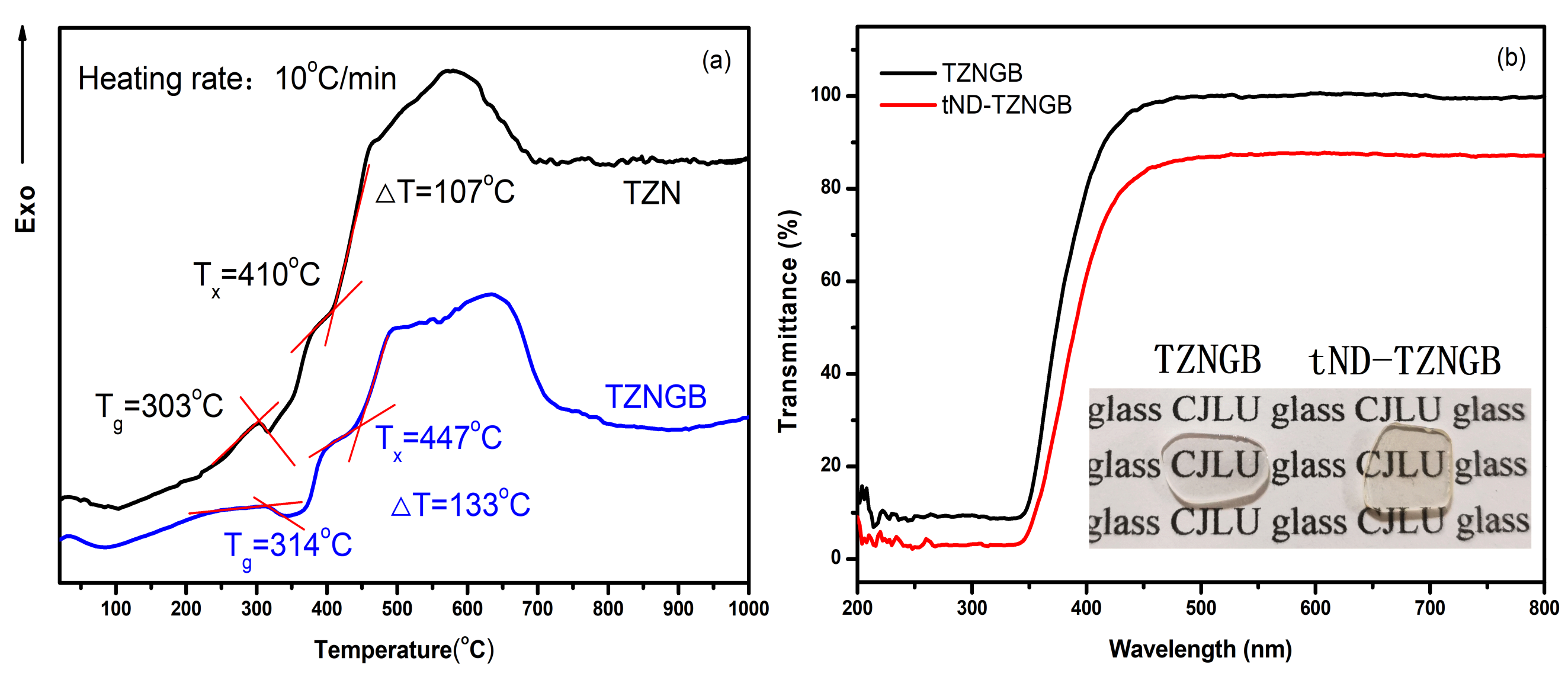




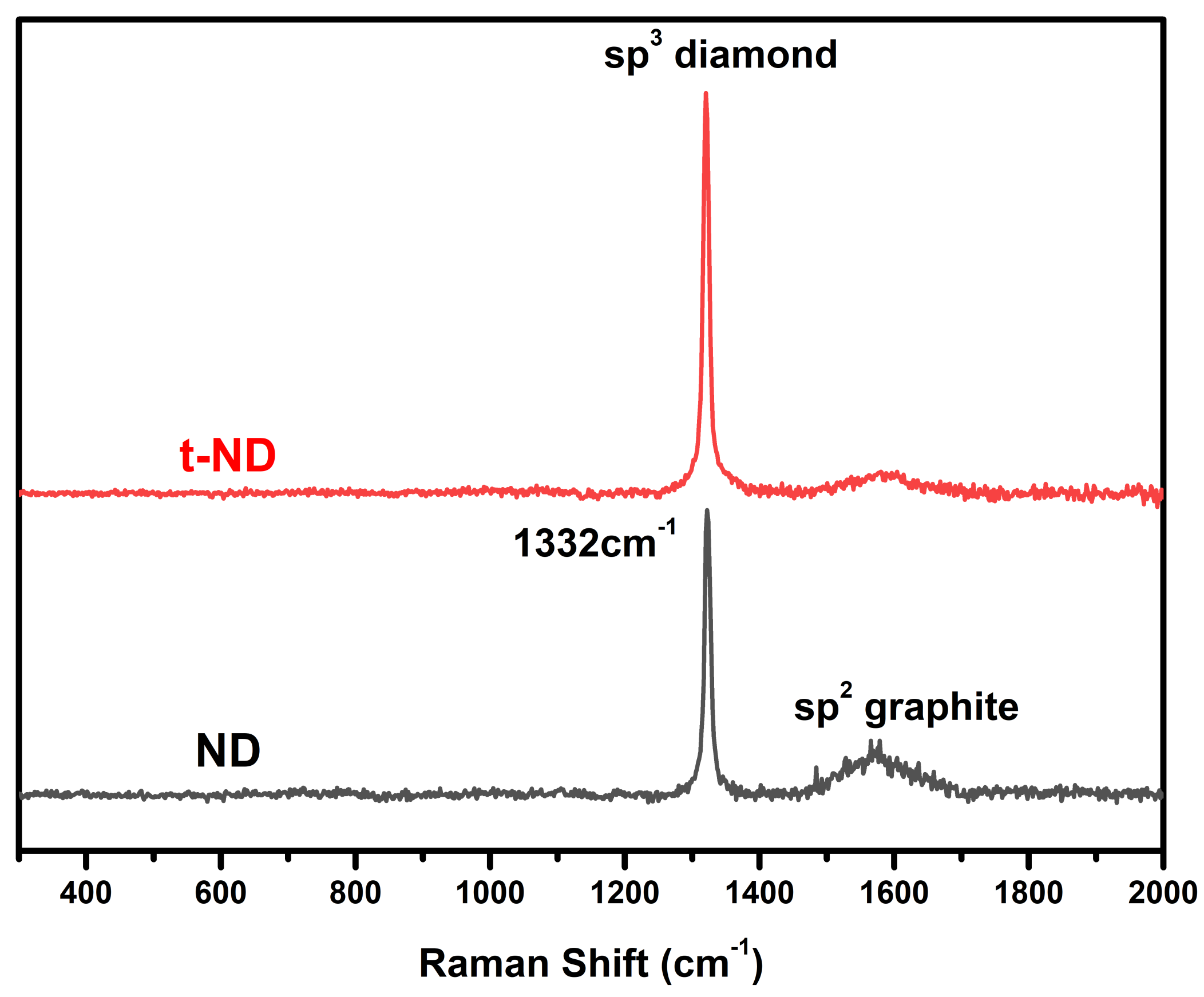




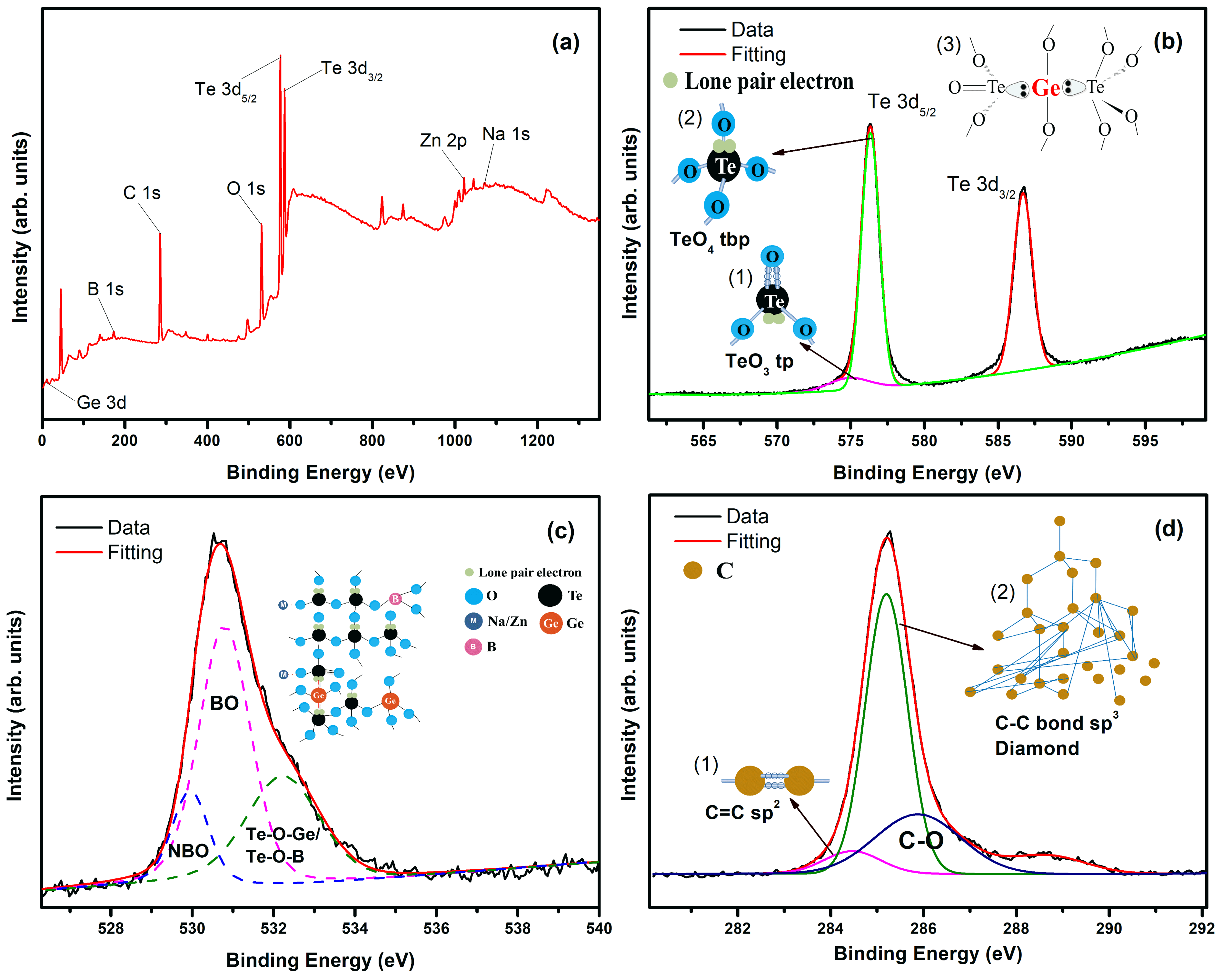

